\title{
Hopf bifurcation analysis for an epidemic model over the Internet with two delays
}

\section{Tao Zhao* and Dianjie Bi}

\section{*Correspondence:}

zhaotaoaufe@163.com

School of Management Science and

Engineering, Anhui University of

Finance and Economics, Bengbu, China

\begin{abstract}
Taking the delay due to the latent period of computer viruses and the delay due to the period that the anti-virus software uses to clean the computer viruses as the bifurcation parameters, local Hopf bifurcation of an epidemic model over the Internet is studied. We discuss the existence of the Hopf bifurcation under four conditions: (1) $\tau_{1}>0, \tau_{2}=0$, (2) $\tau_{1}=0, \tau_{2}>0$, (3) $\tau_{1}=\tau_{2}=\tau>0$, and (4) $\tau_{1}>0, \tau_{2} \in\left(0, \tau_{20}\right)$. Properties of the Hopf bifurcation about condition (4) are investigated by means of the center manifold theorem and the normal form theory. Finally, some simulations are presented to support our obtained results.
\end{abstract}

Keywords: Computer virus; Hopf bifurcation; Stability; SLBQRS model

\section{Introduction}

The Internet is an indispensable part of our everyday life and it offers us more and more functionalities and facilities. Meanwhile, its rapid development has witnessed the overflow of computer viruses. The epidemic dynamics has been considered as an effective approach to the assessment of the propagation of computer viruses in the Internet [1, 2]. Since the seminal work of Kephart and White [3], many computer virus models, such as SIRS (Susceptible-Infectious-Recovered-Susceptible) models [4-7], SEIRS (Susceptible-Exposed-Infectious-Recovered-Susceptible) models [8-11], SEIQRS (Susceptible-Infectious-Quarantined-Recovered-Susceptible) models [12-14], SVEIR (Susceptible-Vaccinated-Exposed-Infectious-Recovered) models [15-17] and so on, have been proposed by scholars at home and abroad.

However, most of the models above neglect time delays during the spreading process of the computer viruses in the Internet. As is known, time delays may induce Hopf bifurcation and the occurrence of a Hopf bifurcation makes the computer viruses in the Internet be out of control. Some research has been devoted to the computer virus models with time delay. In [5, 6], Ren et al. investigated the Hopf bifurcation of a delayed SIRS computer virus propagation model. In [18], Dong et al. proposed a delayed SEIR (Susceptible-Exposed-Infectious-Recovered) computer virus model with multistate antivirus and studied the dynamical behaviors, which include local asymptotical stability and local Hopf bifurcation, by regarding the time delay as bifurcating parameter. Considering the fact that the recovered computers may be infected again after a temporary immunity period, Zhang and Yang [19] proposed a computer virus model with two delays based on the work in [18] and studied the Hopf bifurcation by regarding the possible combinations

(c) The Author(s) 2018. This article is distributed under the terms of the Creative Commons Attribution 4.0 International License (http://creativecommons.org/licenses/by/4.0/), which permits unrestricted use, distribution, and reproduction in any medium, provided you give appropriate credit to the original author(s) and the source, provide a link to the Creative Commons license, and indicate if changes were made. 
of the two delays as a bifurcation parameter. In addition, many researchers introduced the quarantine strategy into mathematical models and investigated the Hopf bifurcation of the models in order to describe dynamics of computer viruses in a network [20-23]. Recently, Zhang and Wang [24] proposed the following SLBQRS (Susceptible-LatentBreaking-Quarantined-Recovered-Susceptible) computer virus model with latent and breaking-out over the Internet:

$$
\left\{\begin{array}{l}
\frac{d S(t)}{d t}=\mu-\beta S(t)(L(t)+B(t))+\varepsilon R(t)-\mu S(t), \\
\frac{d L(t)}{d t}=\beta S(t)(L(t)+B(t))-(\mu+\alpha) L(t), \\
\frac{d B(t)}{d t}=\alpha L(t)-(\mu+\gamma+\eta+\sigma) B(t), \\
\frac{d Q(t)}{d t}=\gamma B(t)-(\mu+\sigma+\delta) Q(t), \\
\frac{d R(t)}{d t}=\delta Q(t-\tau)-(\mu+\varepsilon) R(t)+\eta B(t-\tau) .
\end{array}\right.
$$

Here, $S(t), L(t), B(t), Q(t)$, and $R(t)$ represent the percentages of the susceptible computer, the latent computer, the breaking computer, the quarantined computer, and the recovered computer at time $t$, respectively. $\mu, \sigma, \alpha, \beta, \gamma, \varepsilon, \eta$, and $\delta$ are the parameters of system (1) and they have the same meanings as those in [24]. $\tau$ is the time delay due to the period that the anti-virus software uses to clean the computer viruses in the breaking and the quarantined computers. Zhang and Wang [24] investigated the effect of the time delay $\tau$ on the stability of system (1).

One of the typical features of the computer viruses in the Internet is their latent characteristic $[5,25]$, and there is a time delay before the latent computers develop themselves into the breaking ones. Thus, we incorporate the time delay due to the latent period of the latent computers in system (1) and consider the following SLBQRS computer virus with two delays:

$$
\left\{\begin{array}{l}
\frac{d S(t)}{d t}=\mu-\beta S(t)(L(t)+B(t))+\varepsilon R(t)-\mu S(t), \\
\frac{d L(t)}{d t}=\beta S(t)(L(t)+B(t))-\mu L(t)-\alpha L\left(t-\tau_{1}\right), \\
\frac{d B(t)}{d t}=\alpha L\left(t-\tau_{1}\right)-(\mu+\gamma+\eta+\sigma) B(t), \\
\frac{d Q(t)}{d t}=\gamma B(t)-(\mu+\sigma+\delta) Q(t), \\
\frac{d R(t)}{d t}=\delta Q\left(t-\tau_{2}\right)-(\mu+\varepsilon) R(t)+\eta B\left(t-\tau_{2}\right),
\end{array}\right.
$$

where $\tau_{1}$ is the time delay due to the latent period of the viruses and $\tau_{2}$ is the time delay due to the period that the anti-virus software uses to clean the computer viruses in the breaking and the quarantined computers.

The structure of this paper is as follows. In the next section, we study the local stability of the viral equilibrium of system (2) and the existence of a local Hopf bifurcation of system (2). In Sect. 3, properties of the Hopf bifurcation are investigated. In Sect. 4, some numerical simulations are presented in order to verify our obtained theoretical results. Section 5 summarizes this work.

\section{Local stability of viral equilibrium and existence of local Hopf bifurcation}

Based on the analysis in [24], we know that system (2) has a unique viral equilibrium $E_{*}\left(S_{*}, L_{*}, B_{*}, Q_{*}, R_{*}\right)$ if $R_{0}=\frac{\beta(\alpha+\gamma+\mu+\eta+\sigma)}{(\alpha+\mu)(\gamma+\mu+\eta+\sigma)}>1$, where

$$
S_{*}=\frac{(\alpha+\mu)(\gamma+\mu+\eta+\sigma)}{\beta(\alpha+\gamma+\mu+\eta+\sigma)}=\frac{1}{R_{0}}, \quad L_{*}=\frac{\gamma+\mu+\eta+\sigma}{\alpha} B_{*},
$$




$$
\begin{aligned}
Q_{*} & =\frac{\gamma}{\mu+\sigma+\delta} B_{*}, \quad R_{*}=\frac{\delta \gamma+\eta(\mu+\sigma+\delta)}{(\mu+\varepsilon)(\mu+\sigma+\delta)} B_{*}, \\
B_{*} & =\frac{\mu \alpha(\mu+\sigma+\delta)(\mu+\varepsilon)\left(1-R_{0}\right)}{R_{0} \alpha \gamma \delta \varepsilon+(\mu+\sigma+\delta)\left[R_{0} \alpha \eta \varepsilon-\beta(\mu+\varepsilon)(\mu+\alpha+\gamma+\eta+\sigma)\right]} .
\end{aligned}
$$

The linearized section of system (2) at $E_{*}\left(S_{*}, L_{*}, B_{*}, Q_{*}, R_{*}\right)$ is

$$
\left\{\begin{array}{l}
\frac{d S(t)}{d t}=a_{11} S(t)+a_{12} L(t)+a_{13} B(t)+a_{15} R(t), \\
\frac{d L(t)}{d t}=a_{21} S(t)+a_{22} L(t)+a_{23} B(t)+b_{22} L\left(t-\tau_{1}\right), \\
\frac{d B(t)}{d t}=a_{33} B(t)+b_{32} L\left(t-\tau_{1}\right), \\
\frac{d Q(t)}{d t}=a_{43} B(t)+a_{44} Q(t), \\
\frac{d R(t)}{d t}=a_{55} R(t)+c_{53} Q\left(t-\tau_{2}\right)+c_{54} B\left(t-\tau_{2}\right),
\end{array}\right.
$$

where

$$
\begin{aligned}
& a_{11}=-\beta\left(L_{*}+B_{*}\right)-\mu, \quad a_{12}=-\beta S_{*}, \quad a_{13}=-\beta S_{*}, \quad a_{15}=\varepsilon, \\
& a_{21}=\beta\left(L_{*}+B_{*}\right), \quad a_{22}=\beta S_{*}-\mu, \quad a_{23}=\beta S_{*}, \\
& a_{33}=-(\mu+\gamma+\sigma+\eta), \quad a_{43}=\gamma, \quad a_{44}=-(\mu+\sigma+\delta), \\
& a_{55}=-(\mu+\varepsilon), \quad b_{22}=-\alpha, \quad b_{32}=\alpha, \quad c_{53}=\eta, \quad c_{54}=\delta .
\end{aligned}
$$

The characteristic equation is

$$
\begin{aligned}
\lambda^{5}+ & A_{4} \lambda^{4}+A_{3} \lambda^{3}+A_{2} \lambda^{2}+A_{1} \lambda+A_{0} \\
& +\left(B_{4} \lambda^{4}+B_{3} \lambda^{3}+B_{2} \lambda^{2}+B_{1} \lambda+B_{0}\right) e^{-\lambda \tau_{1}} \\
& +\left(C_{1} \lambda+C_{0}\right) e^{-\lambda \tau_{2}} \\
= & 0
\end{aligned}
$$

with

$$
\begin{aligned}
A_{0}= & a_{33} a_{44} a_{55}\left(a_{12} a_{21}-a_{11} a_{22}\right), \\
A_{1}= & a_{55}\left[a_{11} a_{22}\left(a_{33}+a_{44}\right)+a_{33} a_{44}\left(a_{11}+a_{22}\right)\right]+a_{11} a_{22} a_{33} a_{44} \\
& -a_{12} a_{21}\left(a_{33} a_{44}+a_{33} a_{55}+a_{44} a_{55}\right), \\
A_{2}= & a_{12} a_{21}\left(a_{33}+a_{44}+a_{55}\right)-a_{11} a_{22}\left(a_{33}+a_{44}\right)-a_{33} a_{44}\left(a_{11}+a_{22}\right) \\
& -a_{55}\left[a_{11} a_{22}+a_{33} a_{44}+\left(a_{11}+a_{22}\right)\left(a_{33}+a_{44}\right)\right], \\
A_{3}= & a_{11} a_{22}+a_{33} a_{44}+\left(a_{11}+a_{22}\right)\left(a_{33}+a_{44}\right)-a_{12} a_{21} \\
& +a_{55}\left(a_{11}+a_{22}+a_{33}+a_{44}\right), \\
A_{4}= & -\left(a_{11}+a_{22}+a_{33}+a_{44}+a_{55}\right), \\
B_{0}= & a_{44} a_{55} b_{32}\left(a_{11} a_{23}-a_{13} a_{21}\right)-a_{11} a_{22} a_{33} a_{44} b_{22}, \\
B_{1}= & a_{13} a_{21} b_{32}\left(a_{44}+a_{55}\right)-a_{23} b_{32}\left(a_{11} a_{44}+a_{11} a_{55}+a_{44} a_{55}\right) \\
& +b_{22}\left[a_{11} a_{22}\left(a_{33}+a_{44}\right)+a_{33} a_{44}\left(a_{11}+a_{22}\right)\right],
\end{aligned}
$$




$$
\begin{aligned}
B_{2}= & a_{23} b_{32}\left(a_{11}+a_{44}+a_{55}\right)-a_{13} a_{21} b_{32} \\
& -b_{22}\left[a_{11} a_{22}+a_{33} a_{44}+\left(a_{11}+a_{22}\right)\left(a_{33}+a_{44}\right)\right], \\
B_{3}= & b_{22}\left(a_{11}+a_{22}+a_{33}+a_{44}\right)-a_{23} b_{32}, \quad B_{4}=-b_{22}, \\
C_{0}= & \left(a_{44} c_{53}-a_{43} c_{54}\right) a_{15} a_{21} b_{32}, \quad C_{1}=-a_{15} a_{21} b_{32} c_{53} .
\end{aligned}
$$

Case 1. $\tau=0$. When $\tau=0$, Eq. (4) becomes

$$
\lambda^{5}+A_{04} \lambda^{4}+A_{03} \lambda^{3}+A_{02} \lambda^{2}+A_{01} \lambda+A_{00}=0,
$$

where

$$
\begin{aligned}
& A_{00}=A_{0}+B_{0}+C_{0}, \quad A_{01}=A_{1}+B_{1}+C_{1}, \\
& A_{02}=A_{2}+B_{2}, \quad A_{03}=A_{3}+B_{3}, \quad A_{04}=A_{4}+B_{4} .
\end{aligned}
$$

Suppose that the following holds:

( $\left.H_{1}\right) A_{00}>0, A_{04}>0, A_{03} A_{04}>A_{02}, A_{02} A_{03} A_{04}>A_{02}^{2}+A_{01} A_{04}^{2}$, and $A_{01} A_{04}\left(2 A_{00}+\right.$ $\left.A_{02} A_{03}\right)+A_{00} A_{02} A_{03}>A_{01}\left(A_{02}^{2}+A_{01} A_{04}^{2}\right)+A_{00}\left(A_{00}+A_{04}^{2} A_{03}^{2}\right)$.

It can be concluded that the viral equilibrium $\left(S_{*}, L_{*}, B_{*}, Q_{*}, R_{*}\right)$ is locally asymptotically stable in the absence of time delay.

Case 2. $\tau_{1}>0, \tau_{2}=0$. Equation (4) becomes

$$
\begin{aligned}
\lambda^{5}+ & A_{24} \lambda^{4}+A_{23} \lambda^{3}+A_{22} \lambda^{2}+A_{21} \lambda+A_{20} \\
& +\left(B_{24} \lambda^{4}+B_{23} \lambda^{3}+B_{22} \lambda^{2}+B_{21} \lambda+B_{20}\right) e^{-\lambda \tau_{1}} \\
& =0
\end{aligned}
$$

where

$$
\begin{aligned}
& A_{20}=A_{0}, \quad A_{21}=A_{1}, \quad A_{22}=A_{2}, \quad A_{23}=A_{3}, \quad A_{24}=A_{4}, \\
& B_{20}=B_{0}+C_{0}, \quad B_{21}=B_{1}+C_{1}, \quad B_{22}=B_{2}, \quad B_{23}=B_{3}, \quad B_{24}=B_{4} .
\end{aligned}
$$

Substituting $\lambda=i \omega_{1}\left(\omega_{1}>0\right)$ into Eq. (6) and separating the real and imaginary parts, one can get that

$$
\left\{\begin{array}{l}
\left(B_{21} \omega_{1}-B_{23} \omega_{1}^{3}\right) \sin \tau_{1} \omega_{1}+\left(B_{24} \omega_{1}^{4}-B_{22} \omega_{1}^{2}+B_{20}\right) \cos \tau_{1} \omega_{1} \\
\quad=A_{22} \omega_{1}^{2}-A_{24} \omega_{1}^{4}-A_{20} \\
\left(B_{21} \omega_{1}-B_{23} \omega_{1}^{3}\right) \cos \tau_{1} \omega_{1}-\left(B_{24} \omega_{1}^{4}-B_{22} \omega_{1}^{2}+B_{20}\right) \sin \tau_{1} \omega_{1} \\
\quad=A_{23} \omega_{1}^{3}-\omega_{1}^{5}-A_{21} \omega_{1}
\end{array}\right.
$$

Adding up the squares of both equations gives

$$
\omega_{1}^{10}+g_{24} \omega_{1}^{8}+g_{23} \omega_{1}^{6}+g_{22} \omega_{1}^{4}+g_{21} \omega_{1}^{2}+g_{20}=0
$$


with

$$
\begin{aligned}
& g_{20}=A_{20}^{2}-B_{20}^{2}, \\
& g_{21}=A_{21}^{2}-2 A_{20} A_{22}-B_{21}^{2}+2 B_{20} B_{22}, \\
& g_{22}=A_{22}^{2}+2 A_{20} A_{24}-2 A_{21} A_{23}-B_{22}^{2}+2 B_{21} B_{23}-2 B_{20} B_{24}, \\
& g_{23}=A_{23}^{2}-2 A_{22} A_{24}+2 A_{21}-B_{23}^{2}+2 B_{22} B_{24}-B_{23}^{2}, \\
& g_{24}=A_{24}^{2}-2 A_{23}-B_{24}^{2} .
\end{aligned}
$$

Let $\omega_{1}^{2}=v_{1}$, then Eq. (8) becomes

$$
v_{1}^{5}+g_{24} v_{1}^{4}+g_{23} v_{1}^{3}+g_{22} v_{1}^{2}+g_{21} v_{1}+g_{20}=0
$$

Based on the discussion in [24], we assume that

$\left(H_{21}\right)$ Eq. (9) has at least one positive root $v_{10}$.

Then Eq. (6) has a pair of purely imaginary roots $\pm i \omega_{10}= \pm i \sqrt{v_{10}}$. For $\omega_{10}$, one has

$$
\tau_{10}=\frac{1}{\omega_{10}} \times \arccos \left\{\frac{h_{21}\left(\omega_{10}\right)}{h_{22}\left(\omega_{10}\right)}\right\},
$$

where

$$
\begin{aligned}
h_{21}\left(\omega_{10}\right)= & \left(B_{23}-A_{24} B_{24}\right) \omega_{10}^{8}+\left(A_{22} B_{24}-A_{23} B_{23}-B_{21}\right) \omega_{10}^{6} \\
& +\left(A_{23} B_{21}+A_{21} B_{23}-A_{20} B_{24}-A_{22} B_{22}-A_{24} B_{20}\right) \omega_{10}^{4} \\
& +\left(A_{20} B_{22}+A_{22} B_{20}-A_{21} B_{21}\right)+\omega_{10}^{2}-A_{20} B_{20}, \\
h_{22}\left(\omega_{10}\right)= & B_{24} \omega_{10}^{8}+\left(B_{23}^{2}-2 B_{22} B_{24}\right) \omega_{10}^{6} \\
& +\left(B_{22}^{2}+2 B_{20} B_{24}-2 B_{21} B_{23}\right) \omega_{10}^{4}+\left(B_{21}^{2}-2 B_{20} B_{22}\right) \omega_{10}^{2}+B_{20}^{2} .
\end{aligned}
$$

Differentiating Eq. (6) with respect to $\tau_{1}$,

$$
\begin{aligned}
{\left[\frac{d \lambda}{d \tau_{1}}\right]^{-1}=} & -\frac{5 \lambda^{4}+4 A_{24} \lambda^{3}+3 A_{23} \lambda^{2}+2 A_{22} \lambda+A_{21}}{\lambda\left(\lambda^{5}+A_{24} \lambda^{4}+A_{23} \lambda^{3}+A_{22} \lambda^{2}+A_{21} \lambda+A_{20}\right)} \\
& +\frac{4 B_{24} \lambda^{3}+3 B_{23} \lambda^{2}+2 B_{22} \lambda+B_{21}}{\lambda\left(B_{24} \lambda^{4}+B_{23} \lambda^{3}+B_{22} \lambda^{2}+B_{21} \lambda+B_{20}\right)}-\frac{\tau_{1}}{\lambda} .
\end{aligned}
$$

This gives

$$
\operatorname{Re}\left[\frac{d \lambda}{d \tau_{1}}\right]_{\tau_{1}=\tau_{10}}^{-1}=\frac{f^{\prime}\left(v_{10}\right)}{\left(B_{21} \omega_{10}-B_{23} \omega_{10}^{3}\right)^{2}+\left(B_{24} \omega_{10}^{4}-B_{22} \omega_{10}^{2}+B_{20}\right)^{2}},
$$

where $v_{10}=\omega_{10}^{2}$ and $f_{1}\left(v_{1}\right)=v_{1}^{5}+g_{24} v_{1}^{4}+g_{23} v_{1}^{3}+g_{22} v_{1}^{2}+g_{21} v_{1}+g_{20}$.

Thus, if $\left(H_{22}\right): f_{1}^{\prime}\left(v_{10}\right) \neq 0$ holds, then $\operatorname{Re}\left[\frac{d \lambda}{d \tau_{1}}\right]_{\tau_{1}=\tau_{10}} \neq 0$. Based on the Hopf bifurcation theorem in [26], we have the following.

Theorem 1 For system (2), if $\left(H_{1}\right),\left(H_{21}\right)$, and $\left(H_{22}\right)$ hold, then $E_{*}\left(S_{*}, L_{*}, B_{*}, Q_{*}, R_{*}\right)$ is asymptotically stable for $\tau_{1} \in\left[0, \tau_{10}\right)$; a Hopf bifurcation occurs at $E_{*}\left(S_{*}, L_{*}, B_{*}, Q_{*}, R_{*}\right)$ when $\tau_{1}=\tau_{10}$. 
Case 3. $\tau_{1}=0, \tau_{2}>0$. Equation (4) becomes

$$
\lambda^{5}+A_{34} \lambda^{4}+A_{33} \lambda^{3}+A_{32} \lambda^{2}+A_{31} \lambda+A_{30}+\left(C_{31} \lambda+C_{30}\right) e^{-\lambda \tau_{2}}=0
$$

where

$$
\begin{array}{ll}
A_{30}=A_{0}+B_{0}, & A_{31}=A_{1}+B-1, \quad A_{32}=A_{2}+B_{2}, \\
A_{33}=A_{3}+B_{3}, & A_{34}=A_{4}+B_{4}, \quad C_{30}=C_{0}, \quad C_{31}=C_{1} .
\end{array}
$$

Let $\lambda=i \omega_{2}\left(\omega_{2}>0\right)$ be a root of Eq. (13). Then

$$
\left\{\begin{array}{l}
C_{31} \omega_{2} \sin \tau_{2} \omega_{2}+C_{30} \cos \tau_{2} \omega_{2}=A_{22} \omega_{2}^{2}-A_{24} \omega_{2}^{4}-p_{0} \\
C_{31} \omega_{2} \cos \tau_{2} \omega_{2}-C_{30} \sin \tau_{2} \omega_{2}=A_{23} \omega_{2}^{3}-\omega_{2}^{5}-A_{21} \omega_{2}
\end{array}\right.
$$

Therefore

$$
\omega_{2}^{10}+g_{34} \omega^{8}+g_{33} \omega^{6}+g_{32} \omega^{4}+g_{31} \omega^{2}+g_{30}=0,
$$

where

$$
\begin{aligned}
& g_{30}=A_{30}^{2}-C_{30}^{2}, \quad g_{31}=A_{31}^{2}-2 A_{30} A_{32}-C_{31}^{2}, \\
& g_{32}=A_{32}^{2}+2 A_{30} A_{34}-2 A_{31} A_{33}, \\
& g_{33}=A_{33}^{2}+2 A_{31}-2 A_{32} A_{34}, \quad g_{34}=A_{34}^{2}-2 A_{33} .
\end{aligned}
$$

Let $\omega^{2}=v_{2}$, Eq. (15) becomes

$$
v_{2}^{5}+g_{34} v_{2}^{4}+g_{33} v_{2}^{3}+g_{32} v_{2}^{2}+g_{31} v_{2}+g_{30}=0
$$

Similar as in Case 2, we assume that

$\left(H_{31}\right)$ Eq. (16) has at least one positive root $v_{20}$.

Then Eq. (13) has a pair of purely imaginary roots $\pm i \omega_{20}= \pm i \sqrt{v_{20}}$. For $\omega_{20}$, we have

$$
\tau_{20}=\frac{1}{\omega_{20}} \times \arccos \left\{\frac{h_{31}\left(\omega_{20}\right)}{h_{32}\left(\omega_{20}\right)}\right\}
$$

where

$$
\begin{aligned}
h_{31}\left(\omega_{20}\right)= & -C_{31} \omega_{20}^{6}+\left(A_{33} C_{31}-A_{34} C_{30}\right) \omega_{20}^{4} \\
& +\left(A_{32} C_{30}-A_{31} C_{31}\right) \omega_{20}^{2}-A_{30} C_{30}, \\
h_{32}\left(\omega_{20}\right)= & C_{31}^{2} \omega_{20}^{2}+C_{30}^{2} .
\end{aligned}
$$

Differentiating both sides of Eq. (13) with respect to $\tau_{2}$, we get

$$
\left[\frac{d \lambda}{d \tau_{2}}\right]^{-1}=-\frac{5 \lambda^{4}+4 A_{34} \lambda^{3}+3 A_{33} \lambda^{2}+2 A_{32} \lambda+A_{31}}{\lambda\left(\lambda^{5}+A_{34} \lambda^{4}+A_{33} \lambda^{3}+A_{32} \lambda^{2}+A_{31} \lambda+A_{30}\right)}+\frac{C_{31}}{\lambda\left(C_{31} \lambda+C_{30}\right)}-\frac{\tau_{2}}{\lambda} .
$$


Thus,

$$
\operatorname{Re}\left[\frac{d \lambda}{d \tau_{2}}\right]_{\tau_{2}=\tau_{20}}^{-1}=\frac{f_{2}^{\prime}\left(v_{20}\right)}{C_{30}^{2}+C_{31}^{2} \omega_{20}^{2}}
$$

where $v_{20}=\omega_{20}^{2}$ and $f_{2}\left(v_{2}\right)=v_{2}^{5}+g_{34} v_{2}^{4}+g_{33} v_{2}^{3}+g_{32} v_{2}^{2}+g_{31} v_{2}+g_{30}$.

Therefore, if $\left(H_{32}\right): f_{2}^{\prime}\left(v_{20}\right) \neq 0$ holds, then $\operatorname{Re}\left[\frac{d \lambda}{d \tau_{2}}\right]_{\tau_{2}=\tau_{20}} \neq 0$. Based on the Hopf bifurcation theorem in [26], we have the following.

Theorem 2 For system (2), if $\left(H_{1}\right),\left(H_{31}\right)$, and $\left(H_{32}\right)$ hold, then $E_{*}\left(S_{*}, L_{*}, B_{*}, Q_{*}, R_{*}\right)$ is asymptotically stable for $\tau_{2} \in\left[0, \tau_{20}\right)$; a Hopf bifurcation occurs at $E_{*}\left(S_{*}, L_{*}, B_{*}, Q_{*}, R_{*}\right)$ when $\tau_{2}=\tau_{20}$.

Case 4. $\tau_{1}=\tau_{2}=\tau>0$. Equation (4) becomes

$$
\begin{aligned}
\lambda^{5}+ & A_{4} \lambda^{4}+A_{3} \lambda^{3}+A_{2} \lambda^{2}+A_{1} \lambda+A_{0} \\
& +\left(B_{4} \lambda^{4}+B_{3} \lambda^{3}+B_{2} \lambda^{2}+B_{1} \lambda+B_{0}\right) e^{-\lambda \tau} \\
& +\left(C_{1} \lambda+C_{0}\right) e^{-2 \lambda \tau} \\
= & 0 .
\end{aligned}
$$

Multiplying by $e^{\lambda \tau}$, Eq. (20) becomes of the following form:

$$
\begin{aligned}
B_{4} \lambda^{4} & +B_{3} \lambda^{3}+B_{2} \lambda^{2}+B_{1} \lambda+B_{0} \\
& +\left(\lambda^{5}+A_{4} \lambda^{4}+A_{3} \lambda^{3}+A_{2} \lambda^{2}+A_{1} \lambda+A_{0}\right) e^{\lambda \tau} \\
& +\left(C_{1} \lambda+C_{0}\right) e^{-\lambda \tau} \\
= & 0 .
\end{aligned}
$$

Assume that $\lambda=i \omega(\omega>0)$ is a root of Eq. (21). Then

$$
\left\{\begin{array}{l}
g_{41}(\omega) \cos \tau \omega-g_{42}(\omega) \sin \tau \omega=g_{43}(\omega) \\
g_{44}(\omega) \sin \tau \omega+g_{45}(\omega) \cos \tau \omega=g_{46}(\omega)
\end{array}\right.
$$

with

$$
\begin{aligned}
& g_{41}(\omega)=A_{4} \omega^{4}-A_{2} \omega^{2}+A_{0}+C_{0}, \quad g_{42}(\omega)=\omega^{5}-A_{3} \omega^{3}+\left(A_{1}-C_{1}\right) \omega, \\
& g_{43}(\omega)=B_{2} \omega^{2}-B_{4} \omega^{4}-B_{0}, \quad g_{44}(\omega)=A_{4} \omega^{4}-A_{2} \omega^{2}+A_{0}-C_{0}, \\
& g_{45}(\omega)=\omega^{5}-A_{3} \omega^{3}+\left(A_{1}+C_{1}\right) \omega, \quad g_{46}(\omega)=B_{3} \omega^{3}-B_{1} \omega .
\end{aligned}
$$

Thus,

$$
\cos \tau \omega=\frac{h_{41}(\omega)}{h_{40}(\omega)}, \quad \sin \tau \omega=\frac{h_{42}(\omega)}{h_{40}(\omega)}
$$


where

$$
\begin{aligned}
h_{40}(\omega)= & \omega^{10}+\left(A_{4}^{2}-2 A_{3}\right) \omega^{8}+\left(A_{3}^{2}-2 A_{2} A_{4}\right) \omega^{6} \\
& +\left(A_{2}^{2}+2 A_{0} A_{4}-2 A_{1} A_{3}\right) \omega^{4}+\left(A_{1}^{2}-C_{1}^{2}-2 A_{0} A_{2}\right) \omega^{2}+A_{0}^{2}-C_{0}^{2}, \\
h_{41}(\omega)= & \left(B_{3}-A_{4} B_{4}\right) \omega^{8}+\left(A_{4} B_{2}+A_{2} B_{4}-A_{3} B_{3}-B_{1}\right) \omega^{6} \\
& +\left(A_{3} B_{1}-A_{2} B_{2}-A_{4} B_{0}+B_{3}\left(A_{1}-C_{1}\right)-B_{4}\left(A_{0}-C_{0}\right)\right) \omega^{4} \\
& +\left(A_{2} B_{0}+B_{2}\left(A_{0}-C_{0}\right)-B_{1}\left(A_{1}-C_{1}\right)\right) \omega^{2}-B_{0}\left(A_{0}-C_{0}\right), \\
h_{42}(\omega)= & B_{4} \omega^{9}+\left(A_{4} B_{3}-A_{3} B_{4}-B_{2}\right) \omega^{7} \\
& +\left(B_{0}+A_{3} B_{2}-A_{4} B_{1}-A_{2} B_{3}+B_{4}\left(A_{1}+C_{1}\right)\right) \omega^{5} \\
& +\left(A_{2} B_{1}-A_{3} B_{0}+B_{3}\left(A_{0}+C_{0}\right)-B_{2}\left(A_{1}+C_{1}\right)\right) \omega^{3} \\
& +\left(B_{0}\left(A_{1}+C_{1}\right)-B_{1}\left(A_{0}+C_{0}\right)\right) \omega .
\end{aligned}
$$

Then

$$
h_{41}^{2}(\omega)+h_{42}^{2}(\omega)=h_{40}^{2}(\omega)
$$

Next, we suppose that

$\left(H_{41}\right)$ Eq. (24) has at least one positive root $\omega_{0}$.

From Eq. (23), we have

$$
\tau_{0}=\frac{1}{\omega_{0}} \times \arccos \left\{\frac{h_{41}\left(\omega_{0}\right)}{h_{40}\left(\omega_{0}\right)}\right\} .
$$

Differentiating Eq. (21) with respect to $\tau$, we get

$$
\left[\frac{d \lambda}{d \tau}\right]^{-1}=\frac{h_{411}(\lambda)}{h_{412}(\lambda)}-\frac{\tau}{\lambda}
$$

where

$$
\begin{aligned}
& h_{411}(\lambda)=4 B_{4} \lambda^{3}+3 B_{3} \lambda^{2}+2 B_{2} \lambda+B_{1}+\left(5 \lambda^{4}+4 A_{4} \lambda^{3}+3 A_{3} \lambda^{2}+2 A_{2} \lambda+A_{1}\right) e^{\lambda \tau}, \\
& h_{412}(\lambda)=\left(C_{1} \lambda^{2}+C_{0} \lambda\right) e^{-\lambda \tau}-\left(\lambda^{6}+A_{4} \lambda^{5}+A_{3} \lambda^{4}+A_{2} \lambda^{3}+A_{1} \lambda^{2}+A_{0} \lambda\right) e^{\lambda \tau} .
\end{aligned}
$$

Further,

$$
\operatorname{Re}\left[\frac{d \lambda}{d \tau}\right]_{\tau=\tau_{0}}^{-1}=\frac{F_{41}\left(\omega_{0}\right) \times F_{43}\left(\omega_{0}\right)+F_{42}\left(\omega_{0}\right) \times F_{44}\left(\omega_{0}\right)}{F_{43}^{2}\left(\omega_{0}\right)+F_{44}^{2}\left(\omega_{0}\right)},
$$

with

$$
\begin{aligned}
F_{41}\left(\omega_{0}\right)= & B_{1}-3 B_{3} \omega_{0}^{2}+\left(5 \omega_{0}^{4}-3 A_{3} \omega_{0}^{2}+A_{1}\right) \cos \tau_{0} \omega_{0} \\
& -\left(2 A_{2} \omega_{0}-4 A_{4} \omega_{0}^{3}\right) \sin \tau_{0} \omega_{0}, \\
F_{42}\left(\omega_{0}\right)= & 2 B_{2} \omega_{0}-4 B_{4} \omega_{0}^{3}+\left(5 \omega_{0}^{4}-3 A_{3} \omega_{0}^{2}+A_{1}\right) \sin \tau_{0} \omega_{0} \\
& +\left(2 A_{2} \omega_{0}-4 A_{4} \omega_{0}^{3}\right) \cos \tau_{0} \omega_{0},
\end{aligned}
$$




$$
\begin{aligned}
F_{43}\left(\omega_{0}\right)= & \left(A_{3} \omega_{0}^{4}-\omega_{0}^{6}-\left(A_{1}-C_{1}\right) \omega_{0}^{2}\right) \cos \tau_{0} \omega_{0} \\
& -\left(A_{4} \omega_{0}^{5}-A_{2} \omega_{0}^{3}+\left(A_{0}-C_{0}\right) \omega_{0}\right) \sin \tau_{0} \omega_{0}, \\
F_{44}\left(\omega_{0}\right)= & \left(A_{3} \omega_{0}^{4}-\omega_{0}^{6}-\left(A_{1}+C_{1}\right) \omega_{0}^{2}\right) \sin \tau_{0} \omega_{0} \\
& +\left(A_{4} \omega_{0}^{5}-A_{2} \omega_{0}^{3}+\left(A_{0}-C_{0}\right) \omega_{0}\right) \cos \tau_{0} \omega_{0} .
\end{aligned}
$$

Based on the Hopf bifurcation theorem in [26], if $\left(H_{42}\right): F_{41}\left(\omega_{0}\right) \times F_{43}\left(\omega_{0}\right)+F_{42}\left(\omega_{0}\right) \times$ $F_{44}\left(\omega_{0}\right) \neq 0$ holds, we have the following.

Theorem 3 For system (2), if $\left(H_{1}\right),\left(H_{41}\right)$, and $\left(H_{42}\right)$ hold, then $E_{*}\left(S_{*}, L_{*}, B_{*}, Q_{*}, R_{*}\right)$ is asymptotically stable for $\tau \in\left[0, \tau_{0}\right)$; a Hopf bifurcation occurs at $E_{*}\left(S_{*}, L_{*}, B_{*}, Q_{*}, R_{*}\right)$ when $\tau=\tau_{0}$.

Case 5. $\tau_{1}>0, \tau_{2}>0$, and $\tau_{2} \in\left(0, \tau_{20}\right)$. Let $\lambda=i \omega_{1}^{*}$ be a root of Eq. (4). Then

$$
\left\{\begin{array}{l}
g_{51}\left(\omega_{1}^{*}\right) \sin \tau_{1} \omega_{1}^{*}+g_{52}\left(\omega_{1}^{*}\right) \cos \tau_{1} \omega_{1}^{*}=g_{53}\left(\omega_{1}^{*}\right), \\
g_{51}\left(\omega_{1}^{*}\right) \cos \tau_{1} \omega_{1}^{*}-g_{52}\left(\omega_{1}^{*}\right) \sin \tau_{1} \omega_{1}^{*}=g_{54}\left(\omega_{1}^{*}\right),
\end{array}\right.
$$

where

$$
\begin{aligned}
& g_{51}\left(\omega_{1}^{*}\right)=B_{1} \omega_{1}^{*}-B_{3}\left(\omega_{1}^{*}\right)^{3}+C_{1} \omega_{1}^{*} \cos \tau_{2} \omega_{1}^{*}-C_{0} \sin \tau_{2} \omega_{1}^{*}, \\
& g_{52}\left(\omega_{1}^{*}\right)=B_{4}\left(\omega_{1}^{*}\right)^{4}-B_{2}\left(\omega_{1}^{*}\right)^{2}+B_{0}+C_{1} \omega_{1}^{*} \sin \tau_{2} \omega_{1}^{*}+C_{0} \cos \tau_{2} \omega_{1}^{*}, \\
& g_{53}\left(\omega_{1}^{*}\right)=A_{2}\left(\omega_{1}^{*}\right)^{2}-A_{4}\left(\omega_{1}^{*}\right)^{4}-A_{0}, \\
& g_{54}\left(\omega_{1}^{*}\right)=A_{3}\left(\omega_{1}^{*}\right)^{3}-\left(\omega_{1}^{*}\right)^{5}-A_{1} \omega_{1}^{*} .
\end{aligned}
$$

Adding up the squares of both equations in Eq. (28) gives

$$
f_{50}\left(\omega_{1}^{*}\right)+f_{51}\left(\omega_{1}^{*}\right) \cos \tau_{2} \omega_{1}^{*}+f_{52}\left(\omega_{1}^{*}\right) \sin \tau_{2} \omega_{1}^{*}=0,
$$

with

$$
\begin{aligned}
f_{50}\left(\omega_{1}^{*}\right)= & \left(\omega_{1}^{*}\right)^{10}+\left(A_{4}^{2}-2 A_{3}-B_{4}^{2}\right)\left(\omega_{1}^{*}\right)^{8} \\
& +\left(A_{3}^{2}+2 A_{1}-2 A_{2} A_{4}-2 B_{2} B_{4}-B_{2}^{2}\right)\left(\omega_{1}^{*}\right)^{6} \\
& +\left(A_{2}^{2}+2 A_{0} A_{4}-2 A_{1} A_{3}-B_{2}^{2}-2 B_{0} B_{4}+2 B_{1} B_{3}\right)\left(\omega_{1}^{*}\right)^{4} \\
& +\left(A_{1}^{2}-2 A_{0} A_{2}+2 B_{0} B_{2}-B_{1}^{2}-C_{1}^{2}\right)\left(\omega_{1}^{*}\right)^{2}+A_{0}^{2}-B_{0}^{2}-C_{0}^{2}, \\
f_{51}\left(\omega_{1}^{*}\right)= & 2\left(\left(B_{3} C_{1}-B_{4} C_{0}\right)\left(\omega_{1}^{*}\right)^{4}+\left(B_{2} C_{0}-B_{1} C_{1}\right)\left(\omega_{1}^{*}\right)^{2}-B_{0} C_{0}\right), \\
f_{52}\left(\omega_{1}^{*}\right)= & -2\left(B_{4} C_{1}\left(\omega_{1}^{*}\right)^{5}-\left(B_{2} C_{1}-B_{3} C_{0}\right)\left(\omega_{1}^{*}\right)^{3}-\left(B_{1} C_{0}-B_{0} C_{1}\right) \omega_{1}^{*}\right) .
\end{aligned}
$$

Similar as in Case 4, we suppose that

$\left(H_{51}\right)$ Eq. (29) has at least one positive root $\omega_{10}^{*}$. Define

$$
\tau_{10}^{*}=\frac{1}{\omega_{10}^{*}} \times \arccos \left\{\frac{g_{51}\left(\omega_{10}^{*}\right) \times g_{54}\left(\omega_{10}^{*}\right)+g_{52}\left(\omega_{10}^{*}\right) \times g_{53}\left(\omega_{10}^{*}\right)}{g_{51}^{2}\left(\omega_{10}^{*}\right)+g_{52}^{2}\left(\omega_{10}^{*}\right)}\right\} .
$$


Differentiating Eq. (4) with respect to $\tau_{1}$, we obtain

$$
\left[\frac{d \lambda}{d \tau_{1}}\right]^{-1}=\frac{h_{511}(\lambda)}{h_{512}(\lambda)}-\frac{\tau_{1}}{\lambda}
$$

where

$$
\begin{aligned}
h_{511}(\lambda)= & 5 \lambda^{4}+4 A_{4} \lambda^{3}+3 A_{3} \lambda^{2}+2 A_{2} \lambda+A_{1} \\
& +\left(4 B_{4} \lambda^{3}+3 B_{3} \lambda^{2}+2 B_{2} \lambda+B_{1}\right) e^{-\lambda \tau_{1}} \\
& +\left(C_{1}-\tau_{2} C_{0}-\tau_{2} C_{1} \lambda\right) e^{-\lambda\left(\tau_{1}+\tau_{2}\right)} \\
h_{512}(\lambda)= & \left(B_{4} \lambda^{5}+B_{3} \lambda^{4}+B_{2} \lambda^{3}+B_{1} \lambda^{2}+B_{0} \lambda\right) e^{-\lambda \tau_{1}} \\
& +\left(C_{1} \lambda^{2}+C_{0} \lambda\right) e^{-\lambda\left(\tau_{1}+\tau_{2}\right)} .
\end{aligned}
$$

Thus,

$$
\operatorname{Re}\left[\frac{d \lambda}{d \tau_{1}}\right]_{\tau_{1}=\tau_{10}^{*}}^{-1}=\frac{F_{51}\left(\omega_{10}^{*}\right) \times F_{53}\left(\omega_{10}^{*}\right)+F_{52}\left(\omega_{10}^{*}\right) \times F_{54}\left(\omega_{10}^{*}\right)}{F_{53}^{2}\left(\omega_{10}^{*}\right)+F_{54}^{2}\left(\omega_{10}^{*}\right)}
$$

with

$$
\begin{aligned}
F_{51}\left(\omega_{10}^{*}\right)= & \left(B_{1}-3 B_{3}\left(\omega_{10}^{*}\right)^{2}+\left(C_{1}-\tau_{2} C_{0}\right) \cos \tau_{2} \omega_{10}^{*}\right. \\
& \left.-\tau_{2} C_{1} \omega_{10}^{*} \sin \tau_{2} \omega_{10}^{*}\right) \cos \tau_{10}^{*} \omega_{10}^{*} \\
& +\left(2 B_{2} \omega_{10}^{*}-4 B_{4}\left(\omega_{10}^{*}\right)^{3}-\left(C_{1}-\tau_{2} C_{0}\right) \sin \tau_{2} \omega_{10}^{*}\right. \\
& \left.-\tau_{2} C_{1} \omega_{10}^{*} \cos \tau_{2} \omega_{10}^{*}\right) \sin \tau_{10}^{*} \omega_{10}^{*}+5\left(\omega_{10}^{*}\right)^{4}-3 A_{3}\left(\omega_{10}^{*}\right)^{2}+A_{1}, \\
F_{52}\left(\omega_{10}^{*}\right)= & \left(2 B_{2} \omega_{10}^{*}-4 B_{4}\left(\omega_{10}^{*}\right)^{3}-\left(C_{1}-\tau_{2} C_{0}\right) \sin \tau_{2} \omega_{10}^{*}\right. \\
& \left.-\tau_{2} C_{1} \omega_{10}^{*} \cos \tau_{2} \omega_{10}^{*}\right) \cos \tau_{10}^{*} \omega_{10}^{*} \\
& -\left(B_{1}-3 B_{3}\left(\omega_{10}^{*}\right)^{2}+\left(C_{1}-\tau_{2} C_{0}\right) \cos \tau_{2} \omega_{10}^{*}\right. \\
& \left.-\tau_{2} C_{1} \omega_{10}^{*} \sin \tau_{2} \omega_{10}^{*}\right) \sin \tau_{10}^{*} \omega_{10}^{*}+2 A_{2} \omega_{10}^{*}-4 A_{4}\left(\omega_{10}^{*}\right)^{3} \\
F_{53}\left(\omega_{10}^{*}\right)= & \left(B_{4}\left(\omega_{10}^{*}\right)^{5}-B_{2}\left(\omega_{10}^{*}\right)^{3}+B_{0} \omega_{10}^{*}+C_{0} \omega_{10}^{*} \cos \tau_{2} \omega_{10}^{*}\right. \\
& \left.+C_{1}\left(\omega_{10}^{*}\right)^{2} \sin \tau_{2} \omega_{10}^{*}\right) \sin \tau_{10}^{*} \omega_{10}^{*} \\
& +\left(B_{3}\left(\omega_{10}^{*}\right)^{4}-B_{1}\left(\omega_{10}^{*}\right)^{2}+C_{0} \omega_{10}^{*} \sin \tau_{2} \omega_{10}^{*}\right. \\
& \left.-C_{1}\left(\omega_{10}^{*}\right)^{2} \cos \tau_{2} \omega_{10}^{*}\right) \cos \tau_{10}^{*} \omega_{10}^{*}, \\
& \left.-C_{1}\left(\omega_{10}^{*}\right)^{2} \cos \tau_{2} \omega_{10}^{*}\right) \sin \tau_{10}^{*} \omega_{10}^{*} \\
& \left.+C_{1}\left(\omega_{10}^{*}\right)^{2} \sin \tau_{2} \omega_{10}^{*}\right) \cos \tau_{10}^{*} \omega_{10}^{*} \\
F_{54}\left(\omega_{10}^{*}\right)= & B_{4}\left(\omega_{10}^{*}\right)^{5}-B_{2}\left(\omega_{10}^{*}\right)^{3}+B_{0} \omega_{10}^{*}+C_{0} \omega_{10}^{*} \cos \tau_{2} \omega_{10}^{*} \\
& B_{1}\left(\omega_{10}^{*}\right)^{2}+C_{0} \omega_{10}^{*} \sin \tau_{2} \omega_{10}^{*} \\
& \\
& \\
&
\end{aligned}
$$

Based on the Hopf bifurcation theorem in [26], if $\left(H_{52}\right): F_{51}\left(\omega_{10}^{*}\right) \times F_{53}\left(\omega_{10}^{*}\right)+F_{52}\left(\omega_{10}^{*}\right) \times$ $F_{54}\left(\omega_{10}^{*}\right) \neq 0$ holds, we have the following. 
Theorem 4 For system (2), if $\left(H_{1}\right),\left(H_{51}\right)$, and $\left(H_{52}\right)$ hold, and $\tau_{2} \in\left(0, \tau_{20}\right)$, then $E_{*}\left(S_{*}, L_{*}\right.$, $\left.B_{*}, Q_{*}, R_{*}\right)$ is asymptotically stable for $\tau \in\left[0, \tau_{10}^{*}\right)$; a Hopf bifurcation occurs at $E_{*}\left(S_{*}, L_{*}, B_{*}\right.$, $\left.Q_{*}, R_{*}\right)$ when $\tau_{1}=\tau_{10}^{*}$.

\section{Properties of the Hopf bifurcation}

In this section, we consider the properties of the Hopf bifurcation when $\tau_{1}>0$ and $\tau_{2} \in$ $\left(0, \tau_{20}\right)$. We assume that $\tau_{2 *}<\tau_{10}^{*}$, where $\tau_{2 *} \in\left(0, \tau_{20}\right)$ in this section. Let $u_{1}(t)=S(t)-S_{*}$, $u_{2}(t)=L(t)-L_{*}, u_{3}(t)=B(t)-B_{*}, u_{4}(t)=Q(t)-Q_{*}, u_{5}(t)=R(t)-R_{*}, \tau_{1}=\tau_{10}^{*}+\mu, \mu \in R$. By the transformation $t=t / \tau_{1}$, system (2) becomes

$$
\dot{u}(t)=L_{\mu} u_{t}+F\left(\mu, u_{t}\right)
$$

where $u_{t}=\left(u_{1}(t), u_{2}(t), u_{3}(t), u_{4}(t), u_{5}(t)\right)^{T}=(S(t), L(t), B(t), Q(t), R(t))^{T} \in R^{5}, u_{t}(\theta)=$ $u(t+\theta) \in C\left([-1,0], R^{5}\right)$, and $L_{\mu}: C \rightarrow R^{5}, F\left(\mu, u_{t}\right) \rightarrow R^{5}$ are defined as follows:

$$
L_{\mu} \phi=\left(\tau_{0}+\mu\right)\left(M_{1} \phi(0)+M_{3} \phi\left(-\frac{\tau_{2 *}}{\tau_{10}^{*}}\right)+M_{2}(-1)\right),
$$

and

$$
F(\mu, \phi)=\left(\begin{array}{c}
-\beta\left(\phi_{1}(0) \phi_{2}(0)+\phi_{1}(0) \phi_{3}(0)\right) \\
\beta\left(\phi_{1}(0) \phi_{2}(0)+\phi_{1}(0) \phi_{3}(0)\right) \\
0 \\
0 \\
0
\end{array}\right)
$$

with

$$
\begin{aligned}
M_{1} & =\left(\begin{array}{ccccc}
a_{11} & a_{12} & a_{13} & 0 & a_{15} \\
a_{21} & a_{22} & a_{23} & 0 & 0 \\
0 & 0 & a_{33} & 0 & 0 \\
0 & 0 & a_{43} & a_{44} & 0 \\
0 & 0 & 0 & 0 & a_{55}
\end{array}\right), M_{2}=\left(\begin{array}{ccccc}
0 & 0 & 0 & 0 & 0 \\
0 & b_{22} & 0 & 0 & 0 \\
0 & b_{32} & 0 & 0 & 0 \\
0 & 0 & 0 & 0 & 0 \\
0 & 0 & 0 & 0 & 0
\end{array}\right), \\
M_{3} & =\left(\begin{array}{ccccc}
0 & 0 & 0 & 0 & 0 \\
0 & 0 & 0 & 0 & 0 \\
0 & 0 & 0 & 0 & 0 \\
0 & 0 & 0 & 0 & 0 \\
0 & 0 & c_{53} & c_{54} & 0
\end{array}\right) .
\end{aligned}
$$

Then there exists a function $\eta(\theta, \mu)$ in $\theta \in[-1,0]$ such that

$$
L_{\mu} \phi=\int_{-1}^{0} d \eta(\theta, \mu) \phi(\theta), \quad \phi \in C
$$


In fact,

$$
\eta(\theta, \mu)= \begin{cases}\left(\tau_{10}^{*}+\mu\right)\left(M_{1}+M_{2}+M_{3}\right), & \theta=0, \\ \left(\tau_{10}^{*}+\mu\right)\left(M_{2}+M_{3}\right), & \theta \in\left[-\frac{\tau_{2 *}}{\tau_{10}^{*}}, 0\right), \\ \left(\tau_{10}^{*}+\mu\right) M_{3}, & \theta \in\left(-1,-\frac{\tau_{2 *}}{\tau_{10}^{*}}\right), \\ 0, & \theta=-1 .\end{cases}
$$

For $\phi \in C\left([-1,0], R^{5}\right)$, we set

$$
A(\mu) \phi= \begin{cases}\frac{d \phi(\theta)}{d \theta}, & -1 \leq \theta<0 \\ \int_{-1}^{0} d \eta(\theta, \mu) \phi(\theta), & \theta=0\end{cases}
$$

and

$$
R(\mu) \phi= \begin{cases}0, & -1 \leq \theta<0, \\ F(\mu, \phi), & \theta=0 .\end{cases}
$$

Then system (33) becomes

$$
\dot{u}(t)=A(\mu) u_{t}+R(\mu) u_{t}
$$

For $\varphi \in C^{1}\left([0,1],\left(R^{5}\right)^{*}\right)$, define

$$
A^{*}(\varphi)= \begin{cases}-\frac{d \varphi(s)}{d s}, & 0<s \leq 1, \\ \int_{-1}^{0} d \eta^{T}(s, 0) \varphi(-s), & s=0,\end{cases}
$$

and define

$$
\langle\varphi(s), \phi(\theta)\rangle=\bar{\varphi}(0) \phi(0)-\int_{\theta=-1}^{0} \int_{\xi=0}^{\theta} \bar{\varphi}(\xi-\theta) d \eta(\theta) \phi(\xi) d \xi
$$

where $\eta(\theta)=\eta(\theta, 0)$.

According to the discussion above, $A(0)$ and $A^{*}(0)$ are adjoint operators. And $\pm i \omega_{10}^{*}$ are the eigenvalues of $A(0)$; they are also the eigenvalues of $A^{*}(0)$.

Let $q(\theta)=\left(1, q_{2}, q_{3}, q_{4}, q_{5}\right)^{T} e^{i \omega_{10}^{*} \tau_{10}^{*} \theta}$ and $q^{*}(s)=\left(1, q_{2}^{*}, q_{3}^{*}, q_{4}^{*}, q_{5}^{*}\right) e^{i \omega_{10}^{*} \tau_{10}^{*} s}$ be the eigenvector of $A(0)$ and $A^{*}(0)$ corresponding to $+i \omega_{10}^{*} \tau_{10}^{*}$ and $-i \omega_{10}^{*} \tau_{10}^{*}$, respectively. Then

$$
\begin{aligned}
& q_{2}=\frac{a_{21}+a_{23} q_{3}}{i \omega_{10}^{*}-a_{22}-b_{22} e^{-i \tau \tau_{10}^{*} \omega_{10}^{*}}}, \quad q_{3}=\frac{b_{32} e^{-i \tau_{10}^{*} \omega_{10}^{*}}}{i \omega_{10}^{*}-a_{33}}, \\
& q_{4}=\frac{\left(i \omega_{10}^{*}-a_{55}\right) q_{5}-c_{53} e^{-i \tau_{2 *} \omega_{10}^{*}}}{c_{54} e^{-i \tau_{2 *} \omega_{10}^{*}}}, \quad q_{5}=\frac{i \omega_{10}^{*}-a_{11}-a_{12} q_{2}-a_{13} q_{3}}{a_{15}}, \\
& q_{2}^{*}=-\frac{i \omega_{10}^{*}+a_{11}}{a_{21}}, \quad q_{3}^{*}=-\frac{\left(i \omega_{10}^{*}+a_{22}+b_{22} e^{i \tau_{2 *} \omega_{10}^{*}}\right) q_{2}+a_{12}}{b_{32} e^{i \tau_{2 *} \omega_{10}^{*}}}, \\
& q_{4}^{*}=\frac{c_{54}\left(i \omega_{10}^{*}+a_{55}\right) e^{i \tau_{2 *} \omega_{10}^{*}}}{a_{55}\left(i \omega_{10}^{*}+a_{44}\right)}, \quad q_{5}^{*}=-\frac{i \omega_{10}^{*}+a_{15}}{a_{55}} .
\end{aligned}
$$


Using Eq. (36), we can get

$$
\begin{aligned}
\left\langle q^{*}(s), q(\theta)\right\rangle= & \bar{D}\left[1+q_{2} \bar{q}_{2}^{*}+q_{3} \bar{q}_{3}^{*}+q_{4} \bar{q}_{4}^{*}+q_{5} \bar{q}_{5}^{*}\right. \\
& \left.+\tau_{10}^{*} e^{-i \tau_{10}^{*} \omega_{10}^{*}} q_{2}\left(b_{22} \bar{q}_{2}^{*}+b_{32} \bar{q}_{3}^{*}\right)+\tau_{2 *} e^{-i \tau_{2 *} \omega_{10}^{*}} q_{5}^{*}\left(c_{53} q_{3}+c_{54} q_{4}\right)\right] .
\end{aligned}
$$

We choose

$$
\begin{aligned}
\bar{D}= & {\left[1+q_{2} \bar{q}_{2}^{*}+q_{3} \bar{q}_{3}^{*}+q_{4} \bar{q}_{4}^{*}+q_{5} \bar{q}_{5}^{*}\right.} \\
& \left.+\tau_{10}^{*} e^{-i \tau_{10}^{*} \omega_{10}^{*}} q_{2}\left(b_{22} \bar{q}_{2}^{*}+b_{32} \bar{q}_{3}^{*}\right)+\tau_{2 *} e^{-i \tau_{2 *} \omega_{10}^{*}} q_{5}^{*}\left(c_{53} q_{3}+c_{54} q_{4}\right)\right]^{-1}
\end{aligned}
$$

such that $\left\langle q^{*}, q\right\rangle=1$ and $\left\langle q^{*}, \bar{q}\right\rangle=0$.

Following the algorithms introduced in [26] and using a computation process similar to that in [27-29], we obtain

$$
\begin{aligned}
g_{20}= & 2 \tau_{10}^{*} \bar{D} \beta\left(\bar{q}_{2}^{*}-1\right)\left(q_{2}+q_{3}\right), \\
g_{11}= & \tau_{10}^{*} \bar{D} \beta\left(\bar{q}_{2}^{*}-1\right)\left(\operatorname{Re}\left\{q_{2}\right\}+\operatorname{Re}\left\{q_{3}\right\}\right), \\
g_{02}= & 2 \tau_{10}^{*} \bar{D} \beta\left(\bar{q}_{2}^{*}-1\right)\left(\bar{q}_{2}+\bar{q}_{3}\right), \\
g_{21}= & 2 \beta \tau_{10}^{*} \bar{D}\left(\bar{q}_{2}^{*}-1\right)\left(W_{11}^{(1)}(0) q_{2}+\frac{1}{2} W_{20}^{(1)}(0) \bar{q}_{2}+W_{11}^{(2)}(0)+\frac{1}{2} W_{20}^{(2)}(0)\right. \\
& \left.+W_{11}^{(1)}(0) q_{3}+\frac{1}{2} W_{20}^{(1)}(0) \bar{q}_{3}+W_{11}^{(3)}(0)+\frac{1}{2} W_{20}^{(3)}(0)\right),
\end{aligned}
$$

with

$$
\begin{aligned}
& W_{20}(\theta)=\frac{i g_{20} q(0)}{\tau_{10}^{*} \omega_{10}^{*}} e^{i \tau_{10}^{*} \omega_{10}^{*} \theta}+\frac{i \bar{g}_{02} \bar{q}(0)}{3 \tau_{10}^{*} \omega_{10}^{*}} e^{-i \tau_{10}^{*} \omega_{10}^{*} \theta}+E_{1} e^{2 i \tau_{10}^{*} \omega_{10}^{*} \theta}, \\
& W_{11}(\theta)=-\frac{i g_{11} q(0)}{\tau_{10}^{*} \omega_{10}^{*}} e^{i \tau_{10}^{*} \omega_{10}^{*} \theta}+\frac{i \bar{g}_{11} \bar{q}(0)}{\tau_{10}^{*} \omega_{10}^{*}} e^{-i \tau_{10}^{*} \omega_{10}^{*} \theta}+E_{2},
\end{aligned}
$$

where $E_{1}$ and $E_{2}$ can be determined as follows:

$$
\begin{aligned}
& E_{1}=2\left(\begin{array}{ccccc}
a_{11}^{\prime} & -a_{12} & -a_{13} & 0 & -a_{15} \\
-a_{21} & a_{22}^{\prime} & -a_{23} & 0 & 0 \\
0 & -b_{32} e^{-2 i \tau_{10}^{*} \omega_{10}^{*}} & a_{33}^{\prime} & 0 & 0 \\
0 & 0 & -a_{43} & a_{44}^{\prime} & 0 \\
0 & 0 & -c_{53} e^{-2 i \tau_{2 *} \omega_{10}^{*}} & -c_{54} e^{-2 i \tau_{2 *} \omega_{10}^{*}} & a_{55}^{\prime}
\end{array}\right)^{-1} \\
& \times\left(\begin{array}{c}
-\beta\left(q_{2}+q_{3}\right) \\
\beta\left(q_{2}+q_{3}\right) \\
0 \\
0 \\
0
\end{array}\right),
\end{aligned}
$$




$$
E_{2}=-\left(\begin{array}{ccccc}
a_{11} & a_{12} & a_{13} & 0 & a_{15} \\
a_{21} & a_{22}+b_{22} & a_{23} & 0 & 0 \\
0 & b_{32} & a_{33} & 0 & 0 \\
0 & 0 & a_{43} & a_{44} & 0 \\
0 & 0 & c_{53} & c_{54} & a_{55}
\end{array}\right)^{-1} \times\left(\begin{array}{c}
-\beta\left(\operatorname{Re}\left\{q_{2}\right\}+\operatorname{Re}\left\{q_{3}\right\}\right) \\
\beta\left(\operatorname{Re}\left\{q_{2}\right\}+\operatorname{Re}\left\{q_{3}\right\}\right) \\
0 \\
0 \\
0
\end{array}\right)
$$

where

$$
\begin{aligned}
& a_{11}^{\prime}=2 i \omega_{10}^{*}-a_{11}, \\
& a_{22}^{\prime}=2 i \omega_{10}^{*}-a_{22}-b_{22} e^{-2 i i_{10}^{*} \omega_{10}^{*}} \\
& a_{33}^{\prime}=2 i \omega_{10}^{*}-a_{33}, \\
& a_{44}^{\prime}=2 i \omega_{10}^{*}-a_{44}, \\
& a_{55}^{\prime}=2 i \omega_{10}^{*}-a_{55} .
\end{aligned}
$$

Then we can obtain

$$
\begin{aligned}
& C_{1}(0)=\frac{i}{2 \tau_{10}^{*} \omega_{10}^{*}}\left(g_{11} g_{20}-2\left|g_{11}\right|^{2}-\frac{\left|g_{02}\right|^{2}}{3}\right)+\frac{g_{21}}{2}, \\
& \mu_{2}=-\frac{\operatorname{Re}\left\{C_{1}(0)\right\}}{\operatorname{Re}\left\{\lambda^{\prime}\left(\tau_{10}^{*}\right)\right\}} \\
& \beta_{2}=2 \operatorname{Re}\left\{C_{1}(0)\right\} \\
& T_{2}=-\frac{\operatorname{Im}\left\{C_{1}(0)\right\}+\mu_{2} \operatorname{Im}\left\{\lambda^{\prime}\left(\tau_{10}^{*}\right)\right\}}{\tau_{10}^{*} \omega_{10}^{*}} .
\end{aligned}
$$

In conclusion, we have the following results.

Theorem 5 Let $E_{*}\left(S_{*}, L_{*}, B_{*}, Q_{*}, R_{*}\right)$ be the viral equilibrium of system (2). (i) The Hopf bifurcation at the viral equilibrium $E_{*}\left(S_{*}, L_{*}, B_{*}, Q_{*}, R_{*}\right)$ is supercritical if $\mu_{2}>0$ and subcritical if $\mu_{2}<0$; (ii) the bifurcating periodic solutions are stable if $\beta_{2}<0$ and unstable if $\beta_{2}>0$; (iii) the period of the bifurcating periodic solutions increases if $T_{2}>0$ and decreases if $T_{2}<0$.

\section{Numerical simulations}

In this section, we shall carry out some numerical simulations for illustrating our theoretical analysis. By extracting the same values from [24], we consider the following system:

$$
\left\{\begin{array}{l}
\frac{d S(t)}{d t}=0.02-0.3 S(t)(L(t)+B(t))+0.3 R(t)-0.02 S(t) \\
\frac{d L(t)}{d t}=0.3 S(t)(L(t)+B(t))-0.02 L(t)-0.3 L\left(t-\tau_{1}\right) \\
\frac{d B(t)}{d t}=0.3 L\left(t-\tau_{1}\right)-0.72 B(t) \\
\frac{d Q(t)}{d t}=0.1 B(t)-0.32 Q(t) \\
\frac{d R(t)}{d t}=0.1 Q\left(t-\tau_{2}\right)-0.32 R(t)+0.4 B\left(t-\tau_{2}\right)
\end{array}\right.
$$

By direct computation, using Matlab software package, we get $R_{0}=1.3281$. Then the unique viral equilibrium $E_{*}(0.7530,0.0326,0.0136,0.0043,0.0183)$ is obtained. Also, we know that condition $\left(H_{1}\right)$ is satisfied based on the analysis in [24]. 

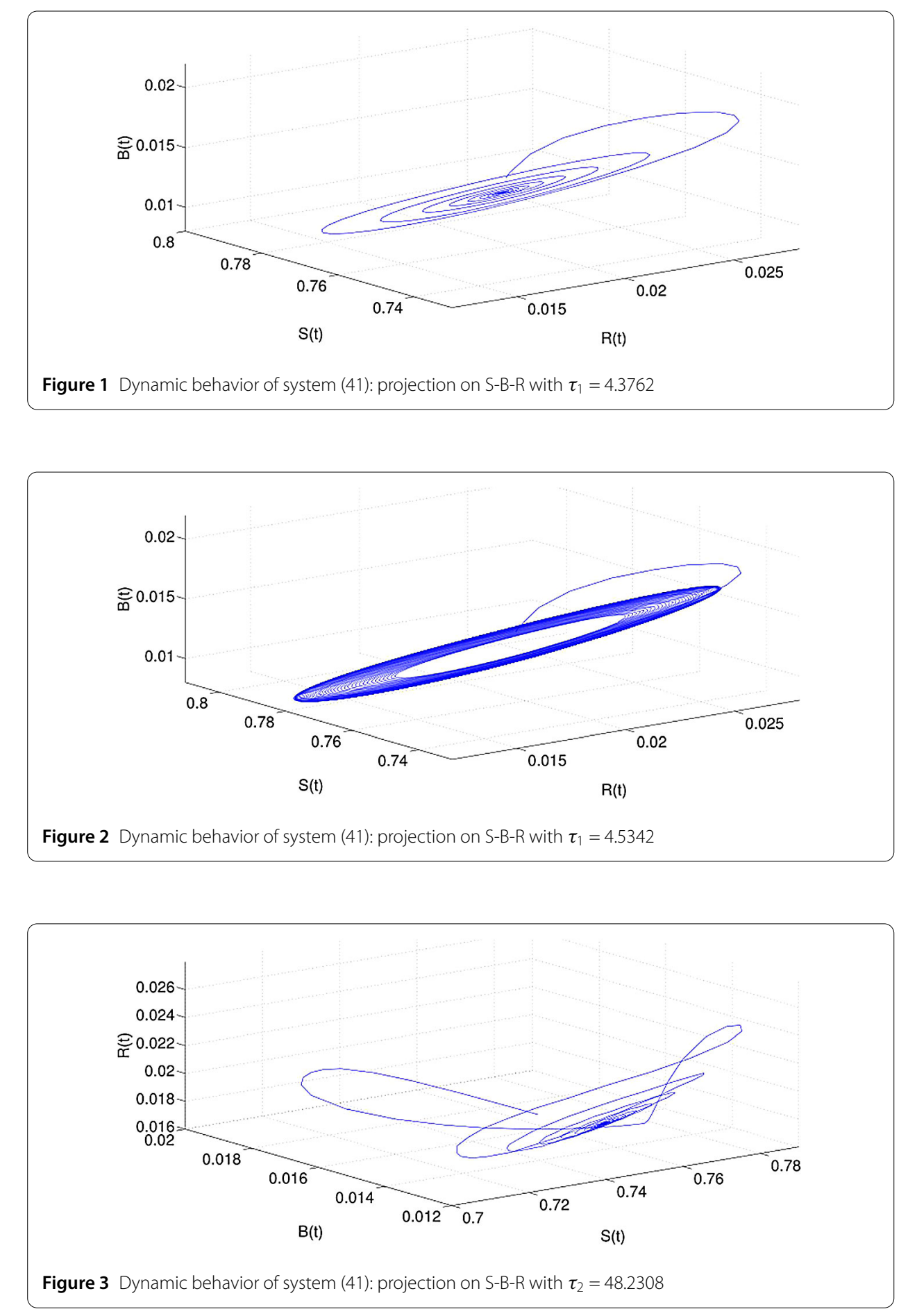

Firstly, one can obtain $\omega_{10}=1.0921, \tau_{10}=4.5186$ by some complicated computations. Based on Theorem 1, we know that the viral equilibrium $E_{*}(0.7530,0.0326,0.0136,0.0043$, $0.0183)$ is asymptotically stable for $\tau_{1}<\tau_{10}=4.5186$. $E_{*}(0.7530,0.0326,0.0136,0.0043$, 0.0183 ) loses its stability and a Hopf bifurcation occurs when $\tau_{1}>\tau_{10}=4.5186$. Simulations can be shown as in Figs. $1-2$. Similarly, we have $\omega_{20}=0.5499, \tau_{20}=53.3616$. The corresponding dynamical behavior of system (41) can be depicted in Figs. 3-4. 


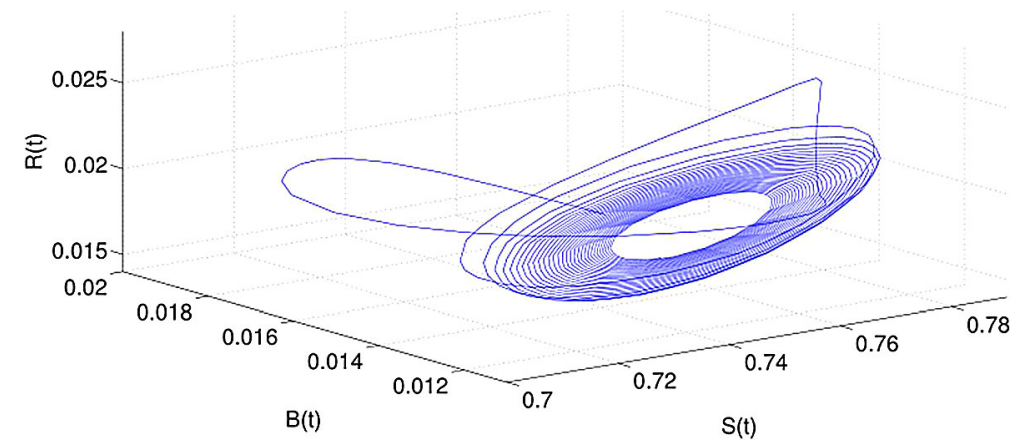

Figure 4 Dynamic behavior of system (41): projection on S-B-R with $\tau_{2}=68.4658$

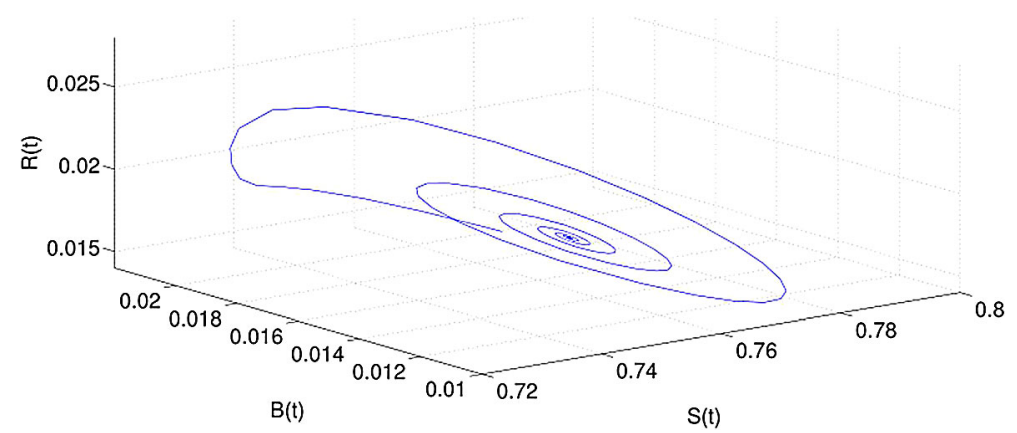

Figure 5 Dynamic behavior of system (41): projection on S-B-R with $\tau=4.0628$

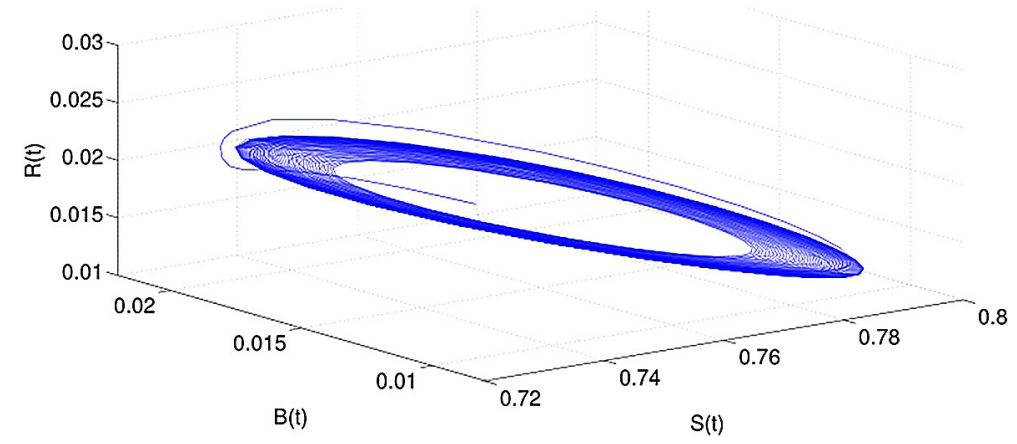

Figure 6 Dynamic behavior of system (41): projection on S-B-R with $\tau=4.3645$

Secondly, by computing, we obtain $\omega_{0}=1.2030, \tau_{0}=4.2686$ for $\tau_{1}=\tau_{2}=\tau>0$. By Theorem 3 , the viral equilibrium $E_{*}(0.7530,0.0326,0.0136,0.0043,0.0183)$ is asymptotically stable for $\tau<\tau_{0}$. However, $E_{*}(0.7530,0.0326,0.0136,0.0043,0.0183)$ becomes unstable and a Hopf bifurcation occurs, when $\tau_{2}$ passes through $\tau_{0}=4.2686$. Dynamical behavior of system (41) in this case can be illustrated by Figs. 5-6.

Lastly, we obtain $\omega_{10}^{*}=2.0254, \tau_{10}^{*}=1.0845$ for $\tau_{1}>0$ and $\tau_{2}=42.5 \in\left(0, \tau_{20}\right)$. As is shown in Fig. 7 , the viral equilibrium $E_{*}(0.7530,0.0326,0.0136,0.0043,0.0183)$ is asymptotically stable when $\tau_{1}=0.8345<\tau_{10}^{*}=1.0845$. However, $E_{*}(0.7530,0.0326,0.0136,0.0043,0.0183)$ will lose its stability and a Hopf bifurcation occurs once $\tau_{1}>\tau_{10}^{*}=1.0845$, which can be 


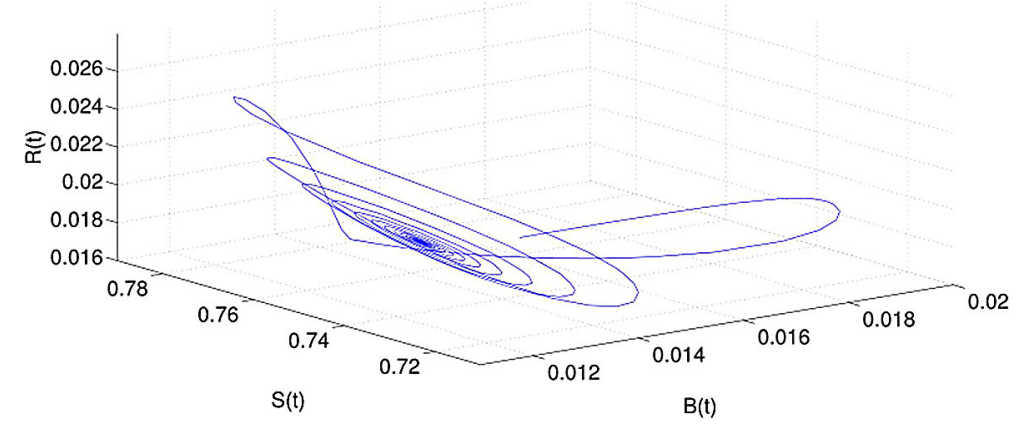

Figure 7 Dynamic behavior of system (41): projection on S-B-R with $\tau_{2}=42.5 \in\left(0, \tau_{20}\right)$ and $\tau_{1}=0.8345$

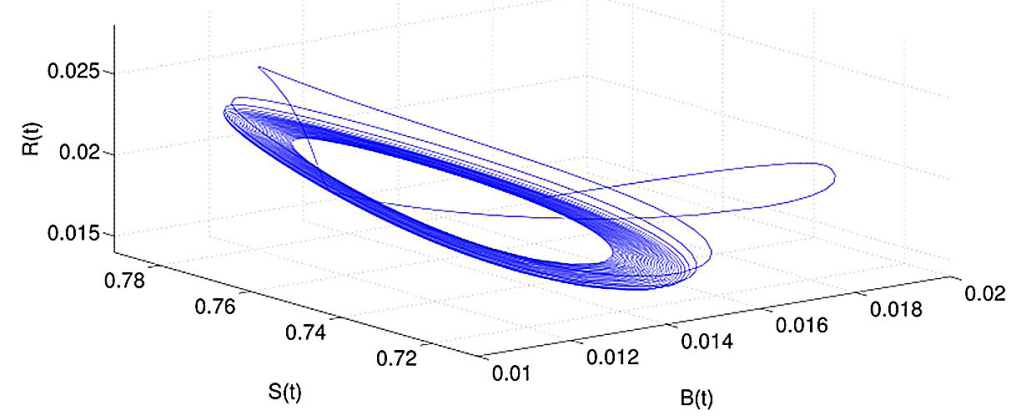

Figure 8 Dynamic behavior of system (41): projection on S-B-R with $\tau_{2}=42.5 \in\left(0, \tau_{20}\right)$ and $\tau_{1}=1.7365$

exhibited by Fig. 8 with $\tau_{1}=1.7365$. Additionally, we obtain $C_{1}(0)=-1.9608+0.3251 i$, $\beta_{2}=-3.9216<0, \mu_{2}=49.6405>0$, and $T_{2}=-6.3854<0$. Based on Theorem 5, we can conclude that the Hopf bifurcation at $\tau_{1}=\tau_{10}^{*}=1.0845$ is supercritical; the bifurcated periodic solutions are stable and the period of the bifurcated periodic solutions decreases.

\section{Conclusions}

An epidemic model over the Internet with two delays is investigated in the present paper by incorporating the time delay due to the latent period of the latent computers in the Internet into the SLBQRS computer virus model considered in the literature [24]. Compared with the model considered in the literature [24], we not only investigate the effects of the time delay due to the latent period of the latent computers, but also the effects of the time delay due to the period that the anti-virus software uses to clean computer viruses in the breaking and the quarantined computers. Obviously, the computer virus model studied in the present paper is more general than that considered in the literature [24].

By taking different combinations of the two delays $\left(\tau_{1}\right.$ and $\left.\tau_{2}\right)$ as the bifurcation parameter, we have successfully studied the local stability of the proposed model and the existence of the local Hopf bifurcation. It is proved that the propagation of the computer viruses can be controlled when the delay is below the corresponding critical value and above which the propagation of the computer viruses will be out of control. For further investigation, the direction of the Hopf bifurcation and the stability and period of the bifurcated periodic solutions are also investigated. However, it should be pointed out that the main purpose of our paper is to investigate the effect of the two delays on system (2), and our study is 
restricted only to the theoretical investigation or experimental studies on the propagation of computer viruses in networks.

Our further research directions include the possibility of linking the results obtained in the present paper with the results coming from the networks theory. Especially, the interest focuses on the impact of the network topology on the propagation of the computer viruses. We leave this as our near future work.

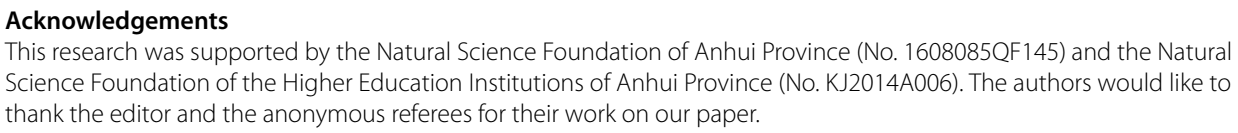

\section{Competing interests}

The authors declare that there are no competing interests regarding the publication of this paper.

\section{Authors' contributions}

All authors read and approved the final manuscript.

\section{Publisher's Note}

Springer Nature remains neutral with regard to jurisdictional claims in published maps and institutional affiliations.

Received: 20 July 2017 Accepted: 27 February 2018 Published online: 15 March 2018

\section{References}

1. Yang, L.X., Yang, X.F., Wu, Y.B.: The impact of patch forwarding on the prevalence of computer virus: a theoretical assessment approach. Appl. Math. Model. 43, 110-125 (2017)

2. Wang, Y., Wen, S., Xiang, Y., et al.: Modeling the propagation of worms in networks: a survey. IEEE Commun. Surv. Tutor. 16, 942-960 (2014)

3. Kephart, J.O., White, S.R.: Directed-graph epidemiological models of computer viruses. In: Proc. 1991 IEEE Comput. Society Symp. Res. Secur. Privacy, pp. 343-359 (1991)

4. Mishra, B.K., Pandey, S.K.: Fuzzy epidemic model for the transmission of worms in computer network. Nonlinear Anal., Real World Appl. 11, 4335-4341 (2010)

5. Ren, J.G., Yang, X.F., Yang, L.X., et al.: A delayed computer virus propagation model and its dynamics. Chaos Solitons Fractals 45, 74-79 (2012)

6. Feng, L.P., Song, L.P., Zhao, Q.S., et al.: Modeling and stability analysis of worm propagation in wireless sensor network. Math. Probl. Eng. 2015, Article ID 129598 (2015)

7. Feng, L.P., Liao, X.F., Li, H.Q., et al.: Hopf bifurcation analysis of a delayed viral infection model in computer networks. Math. Comput. Model. 56, 167-179(2012)

8. Mishra, B.K., Keshri, N.: Mathematical model on the transmission of worms in wireless sensor networks. Appl. Math. Model. 37, 4103-4111 (2013)

9. Mishra, B.K., Pandey, S.K.: Dynamic model of worms with vertical transmission in computer network. Appl. Math. Comput. 217, 8438-8446 (2011)

10. Guillen, J.D.H., Rey, A.M., Encinas, L.H.: Study of the stability of a SEIRS model for computer worm propagation. Physica A 479, 411-421 (2017)

11. Hosseini, S., Azgomi, M.A., Rahmani, A.T.: Malware propagation modeling considering software diversity and immunization. J. Comput. Sci. 13, 49-67 (2016)

12. Wang, F.W., Yang, F., Zhang, Y.K., et al.: Stability analysis of a SEIQRS model with graded infection rates for Internet worms. J. Comput. 9, 2420-2427 (2014)

13. Qaisar, B.: Global stability of SEIQRS computer virus propagation model with non-linear incidence function. Appl. Math. 6, 1926-1938 (2015)

14. Mishra, B.K., Jha, N.: SEIQRS model for the transmission of malicious objects in computer network. Appl. Math. Model. 34, 710-715 (2010)

15. Jia, J., Li, P.: Global analysis of an SVEIR epidemic model with partial immunity. Math. Æterna 8, 547-561 (2011)

16. Wang, F.W., Yang, Y., Zhao, D.M., et al.: A worm defending model with partial immunization and its stability analysis. J. Commun. 10, 276-283 (2015)

17. Upadhyay, R.K., Kumari, S., Misra, A.K.: Modeling the virus dynamics in computer network with SVEIR model and nonlinear incident rate. J. Appl. Math. Comput. 54, 485-509 (2017)

18. Dong, T., Liao, X.F., Li, H.Q.: Stability and Hopf bifurcation in a computer virus model with multistate antivirus. Abstr. Appl. Anal. 2012, Article ID 841987 (2012)

19. Zhang, Z.Z., Yang, H.Z.: Hopf bifurcation analysis for a computer virus model with two delays. Abstr. Appl. Anal. 2013, Article ID 560804 (2013)

20. Liu, J., Bianca, C., Guerrini, L.: Dynamical analysis of a computer virus model with delays. Discrete Dyn. Nat. Soc. 2016, Article ID 5649584 (2016)

21. Yao, Y., Xiang, W.L., Qu, A.D., et al.: Hopf bifurcation in an SEIDQV worm propagation model with quarantine strategy. Discrete Dyn. Nat. Soc. 2012, Article ID 304868 (2012)

22. Yao, Y., Feng, X., Yang, W., et al.: Analysis of a delayed Internet worm propagation model with impulsive quarantine strategy. Math. Probl. Eng. 2014, Article ID 369360 (2014) 
23. Zhang, Z.Z., Song, L.M.: Dynamics of a delayed worm propagation model with quarantine. Adv. Differ. Equ. 2017, Article ID 155 (2017)

24. Zhang, Z.Z., Wang, Y.G.: Qualitative analysis for a delayed epidemic model with latent and breaking-out over the Internet. Adv. Differ. Equ. 2017, Article ID 31 (2017)

25. Kafai, Y.B., White, S.: Understanding virtual epidemics: children's folk conceptions of a computer virus. J. Sci. Educ. Technol. 17, 523-529 (2008)

26. Hassard, B.D., Kazarinoff, N.D., Wan, Y.H.: Theory and Applications of Hopf Bifurcation. Cambridge University Press, Cambridge (1981)

27. Bianca, C., Ferrara, M., Guerrini, L.: The Cai model with time delay: existence of periodic solutions and asymptotic analysis. Appl. Math. Inf. Sci. 7, 21-27 (2013)

28. Bianca, C., Ferrara, M., Guerrini, L.: The time delays' effects on the qualitative behavior of an economic growth model. Abstr. Appl. Anal. 2013, Article ID 901014 (2013)

29. Bianca, C., Guerrini, L.: Existence of limit cycles in the Solow model with delayed-logistic population growth. Sci. World J. 2014, Article ID 207806 (2014)

\section{Submit your manuscript to a SpringerOpen ${ }^{\circ}$} journal and benefit from:

- Convenient online submission

- Rigorous peer review

- Open access: articles freely available online

- High visibility within the field

- Retaining the copyright to your article

Submit your next manuscript at $\gg$ springeropen.com 